\title{
Ultrasound-guided Quadratus Lumborum Block as Postoperative Analgesic Technique for Radical Prostatectomy
}

\author{
${ }^{1}$ Gayathri P Mashar, ${ }^{2}$ Navya Shree, ${ }^{3}$ Yeshaswini Katari, ${ }^{4}$ Hiremathada Sahajananda, ${ }^{5}$ Seema J Mahabaleshwarappaa
}

\begin{abstract}
Postoperative pain after radical retropubic prostatectomy can be severe unless adequately treated, with potential implications for the quality of recovery and length of hospital stay. Ultrasonography-guided quadrates lumborum block (QLB) involves the injection of local anaesthetic solution adjacent to the anterolateral aspect of the quadrates lumborum muscle. QLB is used for postoperative pain relief in abdominal surgeries. We report here a case of $65 \mathrm{yr}$ old male with adenocarcinoma of the prostate gland. He underwent radical retropubic prostatectomy under General anaesthesia with QLB. Post operative analgesia was optimal
\end{abstract}

Keywords: Postoperative analgesia, Quadratus lumborum block, Radical retropubic prostatectomy.

How to cite this article: Mashar GP, Shree N, Katari $Y$, Sahajananda H, Mahabaleshwarappaa SJ. Ultrasound-guided Quadratus Lumborum Block as Postoperative Analgesic Technique for Radical Prostatectomy. J Med Sci 2018;4(1):17-19.

Source of support: Nil

Conflict of interest: None

\section{INTRODUCTION}

Blanco described quadratus lumborum block (QLB) for analgesic in abdominal surgeries. ${ }^{1}$ Currently, QLB is performed as one of the perioperative pain management procedures for pediatric, pregnant, and adult patients undergoing abdominal surgery. ${ }^{2}$ The QLB is an abdominal truncal block for analgesia after abdominal surgery and is known to alleviate somatic pain in both upper and lower abdomen. The QLB performed uses a fascial compartment path to extend the distribution of local anesthesia into the abdominal wall and paravertebral space. This effect can be of vital importance when managing the visceral pain after laparotomies.

\footnotetext{
${ }^{1-3}$ Resident, ${ }^{4}$ Professor and Head, ${ }^{5}$ Assistant Professor

${ }^{1-5}$ Department of Anesthesiology, RajaRajeswari Medical College \& Hospital, Bengaluru, Karnataka, India

Corresponding Author: Gayathri P Mashar, Resident Department of Anesthesiology, RajaRajeswari Medical College \& Hospital, Bengaluru, Karnataka, India, Phone: +918880779309 e-mail: mashar0101@gmail.com
}

The QLB in single shot has the advantage of covering all the dermatomal segments from T4 cranially to L2 segments caudally as the drug is expected to travel from the quadratus lumborum $(\mathrm{QL})$ to the higher vertebral spaces. Midline incision requires bilateral blocks for adequate coverage. Quadratus lumborum block can be used for adequate analgesia in abdominal surgeries, such as exploratory laparotomy, large bowel resection, ileostomy, open/laparoscopic appendectomy, and cholecystectomy; obstetric surgeries, such as cesarean section, total abdominal hysterectomy; urological surgeries, such as open prostatectomy, renal transplant surgery, and nephrectomy.

\section{SONO ANATOMY OF THE QUADRATUS LUMBORUM}

After recognizing three layers of abdominal wall muscles, transversus abdominis is traced more posteriorly until the transversus aponeurosis appears. At this region, usually we can find the peritoneum curves away from the muscles from anterior to posterior and the retroperitoneal fat lies behind the peritoneum and deep to the transversalis fascia. The retroperitoneal fat is generally scanty above the iliac crest and more prominent closer to the iliac crest. Tilting the probe slightly caudal into the pelvis thus improves the view of the retroperitoneal fat and the tapered end of transversus aponeurosis. Quadratus lumborum is usually identified medial to the aponeurosis of transversus abdominis muscle. ${ }^{3}$

Effective postoperative analgesia after open abdominal surgeries is important because it enables early ambulation and helps in managing acute pain which is the most common complaint in patients undergoing these major surgeries. Pain after abdominal surgery is often treated inadequately and maximum utilization of the available resources is essential for improving pain management. Several case reports have shown that local anesthetic injection around the QL muscle is effective in providing pain relief after various abdominal operations and in patients with chronic pain.

\section{CASE REPORT}

A 65-year-old retired businessman initially complained of frequency of micturition, urinary urgency, and 
hesitancy associated with a weak stream. Over the past several weeks, he has reported a few episodes of hematuria and incontinence. In addition to his urologic symptoms, the patient complained of low-grade, constant back pain, and bouts of constipation. A digital rectal examination revealed the patient had an enlarged prostate gland. His past medical history revealed that he was recently diagnosed hypertensive on regular treatment with Tab. Clonidine $10 \mathrm{mg}$ plus Telmisartan $40 \mathrm{mg}$ once daily. The patient had a prostate-specific antigen level of $25 \mathrm{ng} / \mathrm{mL}(0.0-4.0 \mathrm{ng} / \mathrm{mL})$. His hemoglobin was $14.6 \mathrm{gm}$, hematocrit $44 \%$, white blood cell $8,840 / \mathrm{mm}^{3}$, normal differential count, platelets 269,000 / $\mathrm{mm}^{3}$, blood urea nitrogen $23 \mathrm{mg} / \mathrm{dL}$, and creatinine level $1.3 \mathrm{mg} / \mathrm{dL}$. Alkaline phosphatase and liver function tests were all within normal range. Histologic evaluation of the Transurethral Resection of Bladder Tumor biopsy specimen revealed adenocarcinoma, representing $70 \%$ of the biopsied material. An open radical retro pubic prostatectomy was planned for this patient. General anesthesia with QLB was planned for this patient. Patient was shifted to operating theater and all the physiological monitors were attached. His baseline vitals were heart rate: $88 \mathrm{bpm}$, blood pressure: $130 / 80 \mathrm{~mm} \mathrm{Hg}$, and $\mathrm{SpO}_{2} 100 \%$. The patient was premedicated with injection Glycopyrrolate $0.2 \mathrm{mg}$ intravenous (IV) and injection Midazolam $1 \mathrm{mg}$ IV. The patient was preoxygenated with $100 \% \mathrm{O}_{2}$ for 3 minutes. Then he was induced with injection Etomidate $20 \mathrm{mg}+$ injection Fentanyl $100 \mu$ g. After injection Vecuronium $8 \mathrm{mg}$, the patient was intubated with a cuffed endotracheal tube of size $8 \mathrm{~mm}$, bilateral air entry was checked, and tube was fixed at $19 \mathrm{~cm}$. Anesthesia maintenance was carried out by using Isoflurane along with oxygen and nitrous oxide. Muscle relaxation was continued with injection Vecuronium $1 \mathrm{mg}$ bolus top-up doses.

Patient was positioned in lateral position to obtain appropriate view of QL and transverse abdominis plane (TAP) extensions of lateral abdominal muscles. Aseptic precautions were taken by wearing sterile gown and sterile drapes. Ultrasound machine (Sonosite) with linear probe (5-10 MHz) covered in sterile plastic sheath was placed in the anterior axillary line, midway between the iliac crest and the costal margin to visualize the typical triple abdominal layers (Fig. 1A). Then, the probe was placed in the mid-axillary line and at this juncture, the abdominal layers started to taper. When the probe was placed in the posterior axillary line, the sono anatomy showed the transversus abdominis disappearing and the internal oblique and external oblique muscles forming an aponeurosis and appearance of the QL muscle was noticed (Fig. 1B). At the junction of the tapered ends of abdominal muscles and beginning of QL, an 18-gauge Tuhoy needle was inserted in plane and its position was confirmed by injecting saline. Under ultrasound (US) guidance, saline separating the fascia, similar to what is seen in the TAP block was observed. In that space, 20 $\mathrm{mL}$ of $0.5 \%$ Ropivacaine was injected and this procedure will be repeated on the contralateral side. Once the block is performed on both sides, the patient was put in the lithotomy position and the surgery was started.

Patient remained hemodynamically stable throughout the procedure. Two pints of packed red blood cells were transfused intraoperatively. Injection Paracetamol $1 \mathrm{gm}$ was given intraoperatively. The inhalation agents were cut off at the end of the procedure and the neuromuscular blockade was reversed. Patient was extubated after the extubation criteria was met.

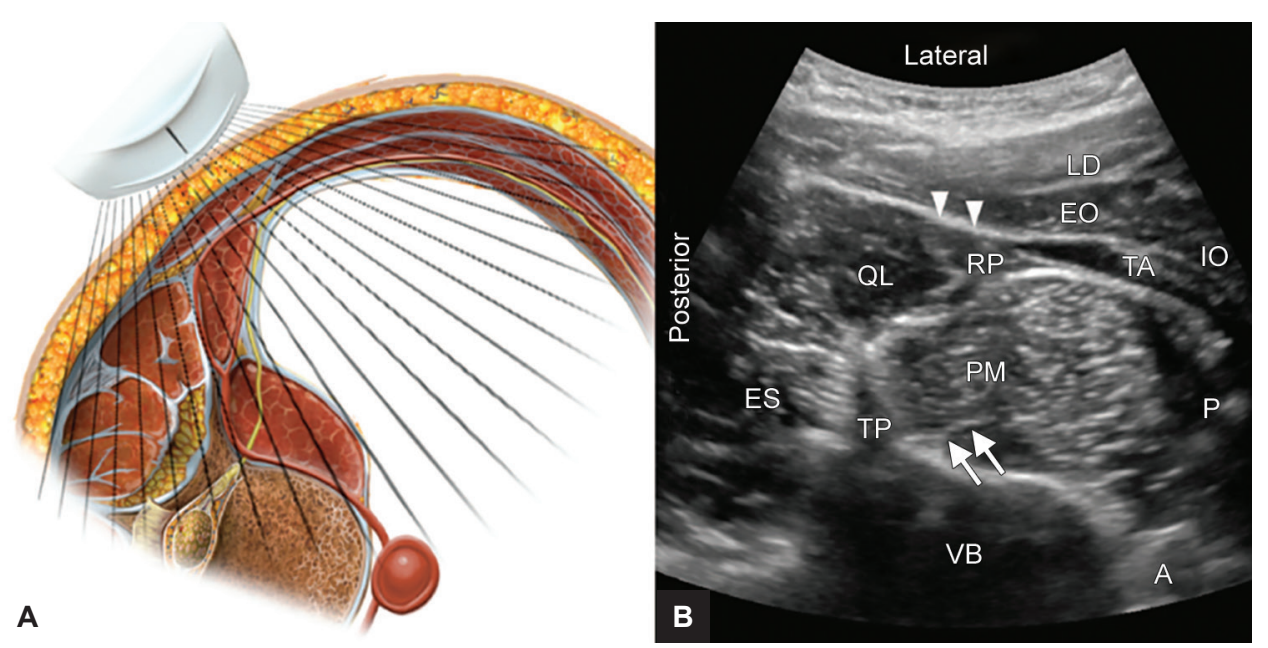

Figs 1A and B: (A) Cross section with the ultrasound probe location. (B) Ultrasound image of the lateral abdominal wall. PM: Psoas major; ES: Erector spinae; TP: Transverse process; VB: Vertebral body (L4); TA: Transverse abdominis; IO: Internal oblique; EO: External oblique; LD: Latissimus dorsi; RP: Retroperitoneal space; P: Peritoneal space; A: Aorta; arrows: lumbar plexus; arrow heads: transversus abdominis aponeurosis 
Patient was shifted to surgical intensive care unit (SICU) postoperatively. In SICU, injection Paracetamol $1 \mathrm{gm}$, 6th hourly and injection Diclofenac $50 \mathrm{mg}$, 12th hourly were administered.

The efficacy of the block was determined by using Numerical Rating Scale (NRS) $(0=$ no pain; $10=$ worst pain imaginable) at rest and on movement. The NRS score on day 1 was 0 and day 2 was 2 . Patient was shifted to surgical ward on 10th postoperative day, and was discharged from the hospital on 12th postoperative day.

\section{DISCUSSION}

This case report shows that QLB has a role in postoperative analgesia in abdominal and pelvic surgeries. In our study case, pain scores were 0 on the 1st day and 2/10 on the 2 nd postoperative day. Patient did not require any opioid in the postoperative period. In this patient, it was easy to perform the unilateral QLB in lateral position in the operation room. We did use bilateral block as the surgeon had planned midline incision. We did require physical support in positioning the patient.

It is said that QLB is the extension of TAP block toward the dorsal region. ${ }^{3}$ According to Hebbard et al, ${ }^{4}$ US-guided TAP block has the limitation of requiring two levels of block to cover incisions above and below umbilicus. The advantage of single-shot QLB is that it covers the dermatome segments from L2 to T4 segments as the drug is expected to travel from the QL to the higher paravertebral spaces. ${ }^{2}$ Carney et $\mathrm{al}^{2}$ described that the contrast solution placed posteriorly accumulates near the lateral border of the QL and then spreads in a posterior-cranial fashion to the anterior aspect of the QL and psoas major to lie at the paravertebral space. They also saw the contrast enhancement from T4 to L2. This was also demonstrated by McDonnell et al ${ }^{5}$ in the landmark technique as a single bolus dose covering the incisions above and below umbilicus. In our case, the sensory block covered T6 to L2. Theoretically, it should be possible as the block spreads to the paravertebral space to block the nerves as they exit. There may be fewer disadvantages to this block.
It may have to be performed in prone position, which is ideal preoperatively, though some of the patients may experience discomfort. If it is done intraoperatively you may need extra personnel to hold the patient and monitor the airway. We should be careful not to cause trauma to kidney. Further, large-scale studies are required to confirm the efficacy of QL block in major abdominal and pelvic surgeries with large midline incisions.

\section{CONCLUSION}

Our case has shown that QLB is useful in postoperative analgesia after major abdominal, pelvic surgery. Largescale randomized controlled trials are necessary to establish its role in routine clinical practice.

\section{ACKNOWLEDGMENTS}

The authors would like to thank Dr Fahim Rajwate and Dr Padmakar, Resident in Urology, Department of Urology, RajaRajeswari Medical College \& Hospital, Bengaluru, Karnataka, India, for allowing them to do the procedure. They also sincerely thank Dr LN Raju, MS MCh, for helping to publish.

\section{REFERENCES}

1. Blanco R. TAP block under ultrasound guidance: the description of a "nonpopstechnique". Reg Anaesth Pain Med 2007;32(Suppl 1):130.

2. Carney J, Finnerty O, Rauf J, Bergin D, Laffey JG, McDonnell GJ. Studies on the spread of local anaesthetic solution in transverses abdominis plane blocks. Anaesthesia 2011 Nov;66(11): 1023-1030

3. Kadam VR. Ultrasound-guided quadratus lumborum block as a postoperative analgesic technique for laparotomy. J Anesthesiol Clin Pharmacol 2013 Oct-Dec'29(4):550-552.

4. Hebbard PD, Barrington MJ, Vasey C. Ultrasound-guided continuous oblique subcostal transversus abdominis plane blockade: description of anatomy and clinical technique. Reg Anesth Pain Med 2010 Sep-Oct;35(5):436-441

5. McDonnell JG, O'Donnell B, Curley G, Heffernan A, Power C, Laffey JG. The analgesic efficacy of transverses abdominis plane block after abdominal surgery: a prospective randomized controlled trial. Anesth Analg 2007 Jan;104(1):193-197 